\title{
Next generation sequencing identified novel heterozygous nonsense mutation in CNGB1 gene associated with retinitis pigmentosa in a Chinese patient
}

\author{
Santasree Banerjee ${ }^{1, *}$, Junping $\mathrm{Yao}^{2, *}$, Xinxin Zhang ${ }^{1, *}$, Jianjun Niu ${ }^{3}$ and Zhongshan \\ Chen ${ }^{4}$ \\ ${ }^{1}$ Department of Cell Biology and Medical Genetics, School of Medicine, Zhejiang University, Hangzhou, China \\ 2 Department of Ophthalmology, Tianyou Hospital Affiliated to Wuhan University of Science \& Technology, Wuhan, China \\ 3 Department of Ophthalmology, General Hospital of Xinjiang Military Area Command of Chinese PLA, Urumqi, China \\ ${ }^{4}$ Department of Ophthalmology, Zhongnan Hospital of Wuhan University, Wuhan, China \\ * These authors contributted equally to this work
}

Correspondence to: Zhongshan Chen, email: czs1112@163.com

Keywords: retinitis pigmentosa, CNGBl gene, midperipheral visual field, rod photoreceptor cells, loss of vision, Pathology Section Received: June 06, $2017 \quad$ Accepted: September 08, $2017 \quad$ Published: October 10, 2017

Copyright: Banerjee et al. This is an open-access article distributed under the terms of the Creative Commons Attribution License 3.0 (CC BY 3.0), which permits unrestricted use, distribution, and reproduction in any medium, provided the original author and source are credited.

\section{ABSTRACT}

Retinitis pigmentosa (RP) is a severe hereditary eye disease characterized by progressive degeneration of photoreceptors and subsequent loss of vision. Retinitis pigmentosa (RP) is a clinically and genetically heterogeneous group of retinal diseases. Germline mutations of CNGB1 is associated with retinitis pigmentosa. We have identified and investigated a 34-year-old Chinese man with markedly have night vision blindness and loss of midperipheral visual field. The proband also lose his far peripheral visual field and also central vision. Proband's retinal pigment deposits visible on fundus examination and primary loss of rod photoreceptor cells followed by secondary loss of cone photoreceptors. Target exome capture based next generation sequencing and Sanger sequencing identified novel nonsense mutation, $C .1917 \mathrm{G}>\mathrm{A}$ and a reported mutation, $c .2361 C>A$, in the CNGB1 gene. Both the nonsense mutations are predicted to lead to the formation of a premature stop codon which finally results into formation of truncated CNGB1 protein product which finally predicted to be disease causing. According to the variant classification guidelines of ACMG, these two variants are categorized as "likely pathogenic" variants. Our findings expand the mutational spectra of CNGB1 and are valuable in the mutation-based pre- and postnatal screening and genetic diagnosis for retinitis pigmentosa.

\section{INTRODUCTION}

Retinitis pigmentosa (RP) is an inherited eye disease with dystrophy of retina dystrophy that finally results into loss of vision. It is genetically and phenotypically extremely heterogenous. According to inheritance pattern, disease onset and clinical symptoms, retinitis pigmentosa is showing extreme heterogeneity. There are almost one and half a million patients with RP, around the world [1]. Patients with RP experienced with degradation of retinal cone and rod cells which finally causes blindness [2]. According to the mode of inheritance, RP has been inherited either dominantly or recessively or with a $\mathrm{X}$-linked pattern [3]. However, very rare cases it has been showed that RP is caused by bigenic pattern. In addition, half of the RP cases are inherited either dominantly $(20 \%)$ or recessively $(20 \%)$ or with a X-linked $(10 \%)$ pattern [4-5]. Due to extreme genetic heterogeneity, genetic screening for RP patients and identifying the causal variants from candidate genes are really a big challenge [6-7].

Based on classical genetics, recessively inherited disease majorly caused by homozygous mutation of a candidate gene in consanguineous families $[8,9]$. 
For such cases, homozygosity mapping is a significant technology for identifying the responsible genes [10]. So, for identifying compound heterozygosity, target exome capture based next generation sequencing technology is the most efficient.

Here, in order to identify the molecular basis of RP in the proband of this Chinese family, we screened a panel of 60 genes associated with retinitis pigmentosa by targeted next-generation sequencing and confirmatory direct sequencing. In this study, we found novel heterozygous nonsense mutations of $C N G B 1$ gene segregating with retinitis pigmentosa phenotype in the proband, with autosomal recessive (compound heterozygosity) inheritance.

\section{RESULTS}

\section{Clinical description}

A 28 years old Chinese man of a nonconsanguineous Chinese family with RP was studied. The pedigree contained only one affected individual (The proband, II-1) (Figure 1). Since childhood, the proband (II-1) has been presented with night blindness with consecutive loss of peripheral vision. During ophthalmic examination (28 years), spicule-shaped pigmentory deposits has been identified in the fundus with progressive reduction of the visual field in both the eyes.

All the family members have given their informed consent for participating in this study.

\section{Identification and characterization of candidate mutation}

We identified two novel nonsense mutation in $C N G B 1$ : c. $1917 \mathrm{G}>\mathrm{A}$ in exon 20 , and c.2361C $>\mathrm{A}$ in exon 24 , inherited from the healthy father and mother respectively (Figure 2). Both of these mutations are predicted to lead to the formation of truncated CNGB1 protein. c. $1917 \mathrm{G}>\mathrm{A}$ and $\mathrm{c} .2361 \mathrm{C}>\mathrm{A}$ leads to the formation of a premature sop codon which in turn predicted to results in premature termination of translation p.Trp639* and p.Tyr $787 *$ respectively. In addition, in silico analysis showed these two novel mutations are potential to cause disease $[11,12]$. These two mutations are classified as "likely pathogenic" variant based on ACMG guidelines [13].

We did not detect these two mutations in the 100 normal control individuals of the same ethnic origin, gender and age range.

\section{DISCUSSION}

In our study, we found two heterozygous lossof-function mutations (c.1917G $>$ A, p.Trp639*; and

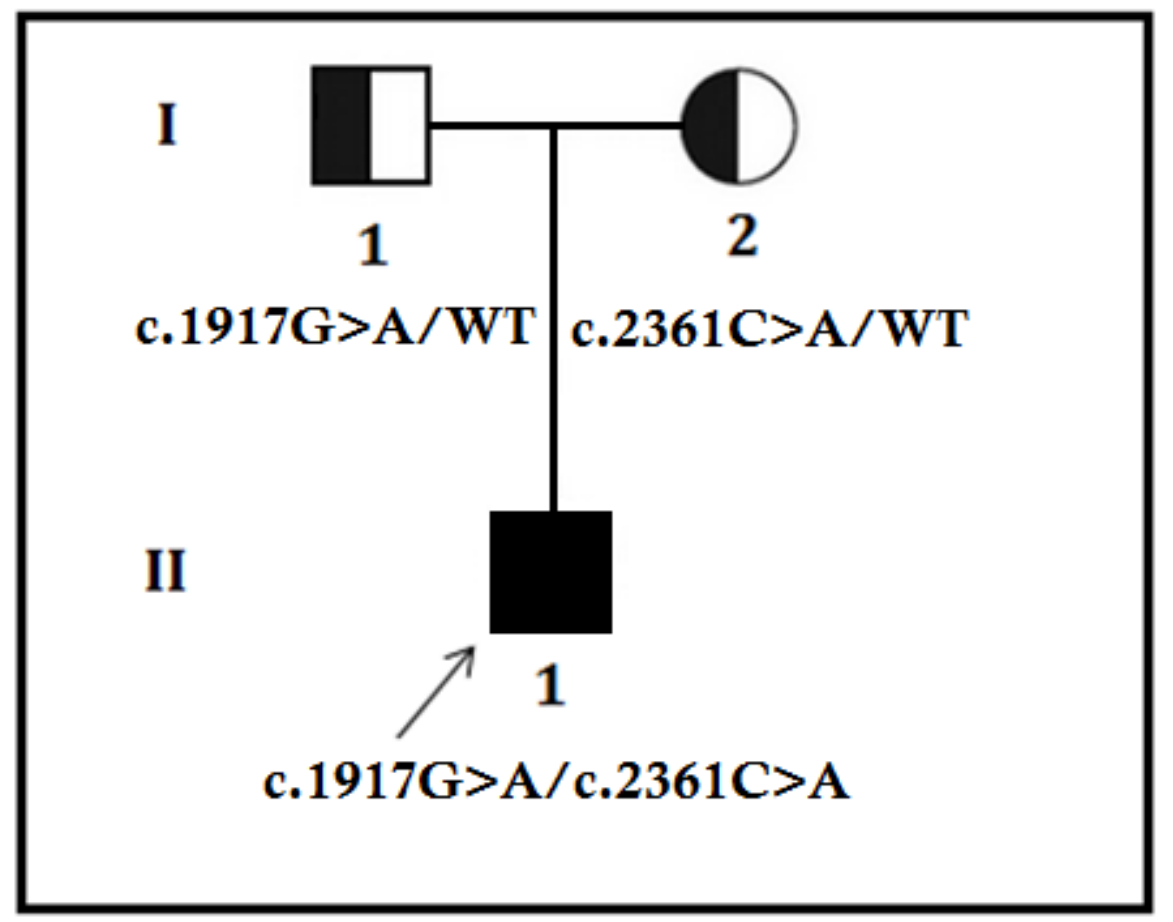

Figure 1: Pedigree of the family. The filled symbol indicates the patient (proband), and the half-filled symbols show the carrier parents, who were heterozygous carriers but were unaffected. The arrow points to the proband. 
c. $2361 \mathrm{C}>\mathrm{A}$, p.Tyr787*) [NCBI Reference sequence NM_001297] of the human CNGB1 gene in the proband (II- $\overline{1})$. Both of these heterozygous nonsense mutations of $C N G B 1$ gene are predicted to form truncated CNGB1 protein in contrast with the wildtype CNGB1 protein consisting of 1251 amino acids. c. $2361 \mathrm{C}>\mathrm{A}$, p. Tyr787* was previously reported by $\mathrm{Xu}$ et al., in 2014 [14].

Germline mutations in $C N G B 1$ genes is associated with autosomal recessive retinitis pigmentosa ( $\operatorname{arRP})$ very rare. Clinical manifestations of our patient are same with all RP patients with mutations $C N G B 1$ gene reported previously.

CNGB1 encode $\beta$-subunits of cyclic nucleotidegated $(\mathrm{CNG})$ channels plays a significant role for signal transduction pathways in both visual and olfactory system. These are ligand-gated channels regulated or controlled directly by second messenger like Cyclic guanosine monophosphate or cyclic adenosine monophosphate. CNG channels control the photo-transduction pathways in photoreceptor cells which is finally leads to photoreceptor cell hyperpolarization [15]. However, CNG channels are composed by a heterotetramer including an $\alpha$-subunit dimer and a $\beta$-subunit dimer [16-19]. Additionally, structurally and functionally $\alpha$ - and $\beta$-subunits are sharing close similarity [20].

Moreover, wild type $\alpha$-subunit and $\beta$-subunit are required for normal function of $\mathrm{CNG}$ channel. Truncating mutation in CNGB1 gene causes formation of non- functional $\mathrm{CNGB1}$ protein which finally affects the normal function of the CNG channel [21, 22].

In conclusion, here, we report a Chinese patient who presented with retinitis pigmentosa, with novel mutations in the $C N G B 1$ gene. Our study is significant for genetic screening and clinical diagnosis of retinitis pigmentosa.

\section{MATERIALS AND METHODS}

\section{Ethical statement}

Proband and his parents of this Chinese family have given written informed consent as they are participating in this study. The Ethical Committee of the Department of Ophthalmology, Zhongnan Hospital of Wuhan University, Wuhan, China, reviewed and approved our study protocol in compliance with the Helsinki declaration. Diagnosis of the patients for retinitis pigmentosa has done by ophthalmologist.

\section{Patients and pedigree}

A proband of Chinese descend with retinitis pigmentosa, diagnosed in the Department of Ophthalmology, Zhongnan Hospital of Wuhan University, Wuhan, China, were enrolled in our study.

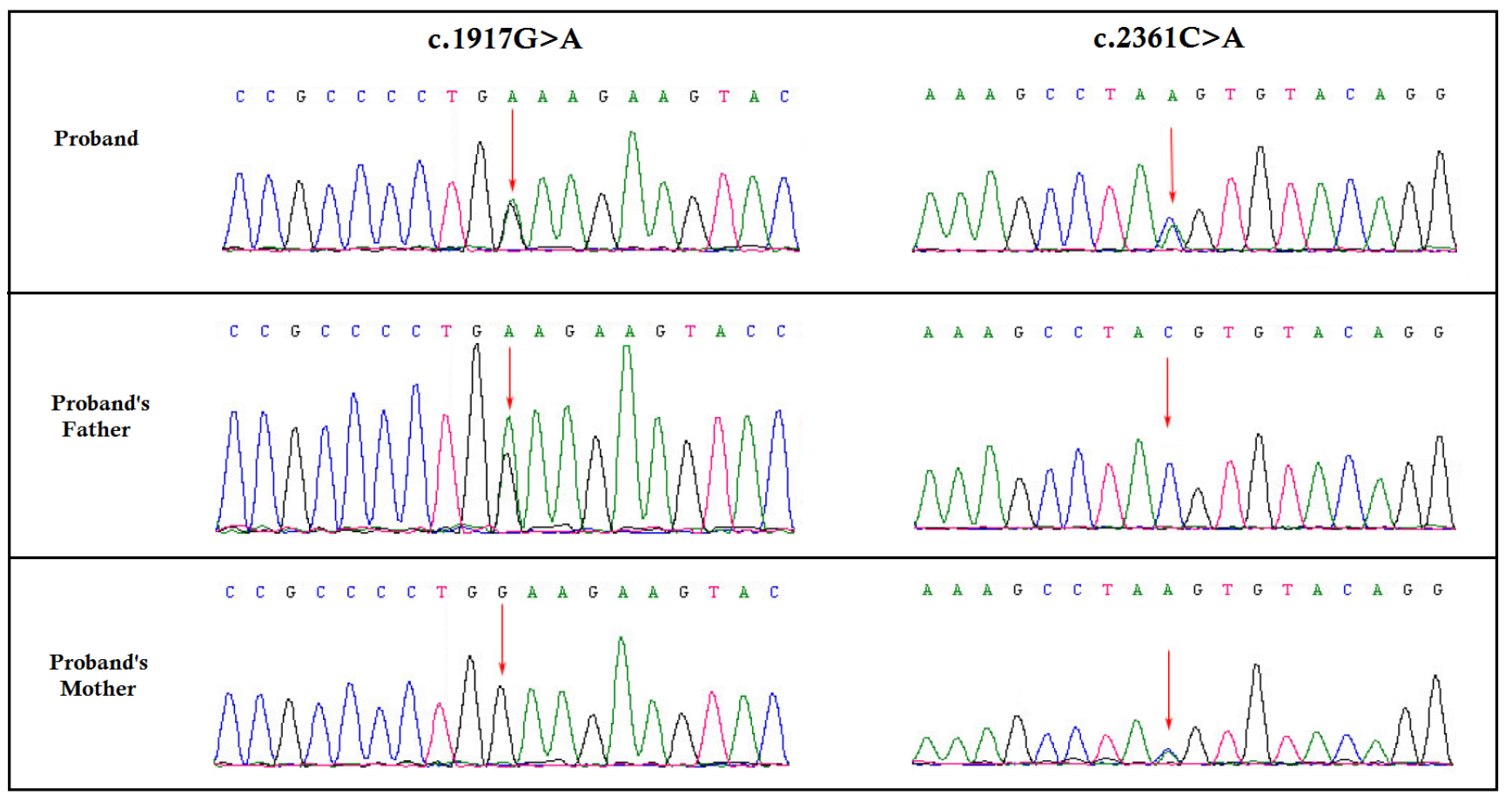

Figure 2: Partial DNA sequences in the CNGB1 by Sanger sequencing of the family [NM_001297]. Upper line: the proband, middle line: the father, bottom line: the mother. Arrows point to the mutations. The proband inherited both $\mathrm{c} .1917 \mathrm{G}>\mathrm{A}$ and c. $2361 \mathrm{C}>$ A mutations. The father carries the $\mathrm{c} .1917 \mathrm{G}>\mathrm{A}$ mutation, and the mother carries the c.2361C $>$ A mutation. 
Targeted exome-based next-generation sequencing and variant identification

DNA samples obtained from the proband (II-1) were sequenced using target exome-based next-generation sequencing. Roche NimbleGen's (Madison,USA) custom Sequence Capture Human Array was used to designed to capture $221340 \mathrm{~kb}$ of targeted sequence, covering 181 exons and flanking sequence (including the $100 \mathrm{bp}$ of introns) of 60 genes (ABCA4, AIPL1, ARL6, BEST1, C2orf71, CA4, CDHR1, CERKL, CLRN1, CNGA1, CNGB1, CRB1, CRX, CYP4V2, DHDDS, EYS, FAM161A, FLVCR1, ,FSCN2, GUCA1B, IDH3B, IMPDH1, IMPG2, KLHL7, LRAT, MAK, MERTK, NR2E3, NRL, OFD1, PDE6A, PDE6B, PDE6G, PRCD, PROM1, PRPF3,

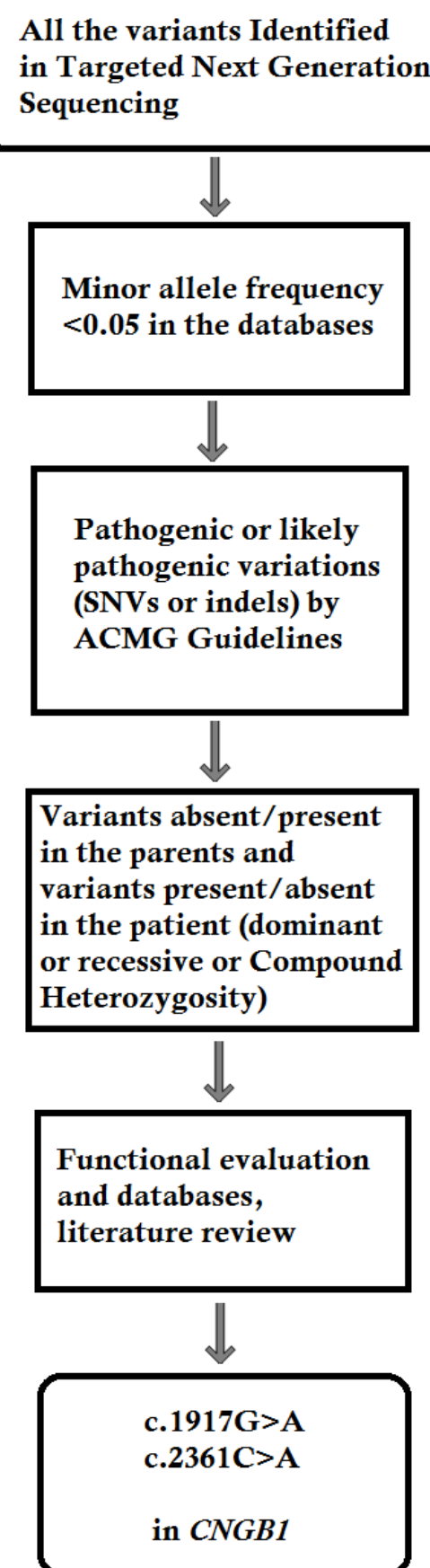

Figure 3: Filtering process for pathogenic mutations in all variations obtained by exome sequencing. * Databases used: dbSNP, Hapmap, 1000 Genomes Project and BGI's in-house database of $\sim 30000$ Chinese people. SNV: single nucleotide variation. Indel: small insertion and deletion. 
PRPF6, PRPF8, PRPF31, PRPH2, RBP3, RDH12, RGR, RHO, RLBP1, ROM1, RP1, RP2, ,RP9, RPE65, RPGR, SAG, SEMA4A, SNRNP200, SPATA7, TOPORS, TTC8, TULP1, USH2A, ZNF513) associated with retinitis pigmentosa and yielded an average of 6366534 reads per sample, with approximately $96.92 \%$ mapping to the targeted regions. The average sequencing depth of the target area is $217.08 \%$ with $99.73 \%$ coverage. The procedure for preparation of libraries was consistent with standard operating protocols. In each pooling batch, 10 to 33 samples were sequenced simultaneously on Illumina HiSeq 2500 Analyzers (Illumina, San Diego, USA) for 90 cycles (specially designed by us for this study). Image analysis, error estimation, and base calling were performed using Illumina Pipeline software (version 1.3.4) to generate raw data. The raw reads were screened to generate - clean reads $\|$ followed by established filtering criteria. Clean reads with a length of $90 \mathrm{bp}$ were aligned to the reference human genome from the NCBI database (Build 37) using the Burrows Wheeler Aligner (BWA) Multi-Vision software package with output files in - bam\| format. The bamdata were used for reads coverage in the target region and sequencing depth computation, SNP and INDEL calling, and CNV detection. First, a novel threestep computational frame work for CNV was applied. Then, SNPs and INDELs were called using SOAPsnp software and Sam tools pileup software, respectively. A SNP or INDEL was be filtered if it could not follow the criterion: supported by at least 10 reads and $>20 \%$ of the total reads. The frequency filter was set at 0.05 . If a SNP frequency was more than 0.05 in any of the four databases (dbSNP, Hapmap, 1000 Genomes Project, the 124 healthy reference samples sequenced in this study), it would be regarded as a polymorphism, but not a causative mutation.

Last, SNVs were retrieved in The Human Gene Mutation Database (http://www.hgmd.cf.ac.uk/ac/index. php) and the Leiden Open Variation Database (http://www. lovd.nl/3.0/home), and then labeled as reported or novel (Figure 3).

\section{Confirmation of the novel splice-site mutation by Sanger sequence}

To validate true positive of the mutation, Sanger sequencing was performed. Primers flanking the candidate loci were designed based on the reference genomic sequences of Human Genome from GenBank in NCBI and synthesized by Invitrogen, Shanghai, China. PCR amplification was carried out in ABI 9700 Thermal Cycler. PCR products were directly sequenced on ABI PRISM 3730 automated sequencer (Applied Biosystems, Foster City, CA, USA). Sequence data comparisons and analysis were performed by DNASTAR SeqMan (DNASTAR, Madison, Wisconsin, USA).

These two heterozygous novel nonsense mutations identified through targeted next generation sequencing were verified through Sanger sequencing using the primers: F1 5'-TTTACCAGTGAGGGACGGGC-3', R1 5'-GTTTGTCTGGCTCCGGTAAGTA-3'. The reference sequence NM_001297 of CNGB1 was used.

\section{Author's contribution}

Santasree Banerjee, Junping Yao, Xinxin Zhang design the study. Xinxin Zhang performed the molecular diagnosis by Sanger sequencing. Santasree Banerjee carried on the bioinformatic analysis. Jianjun Niu, Li Wang contributed to the molecular diagnosis analysis based on NGS. Zhongshan Chen worked on the clinical study. Junping Yao, Xinxin Zhang contributed to the Sanger sequencing validation and quantitative PCR experiments. Santasree Banerjee, Junping Yao, Xinxin Zhang wrote the paper. All authors read and approved the final manuscript.

\section{ACKNOWLEDGMENTS}

We are grateful to our patients for their sincere participation in this study.

\section{CONFLICTS OF INTERESTS}

The authors declare no competing financial interests.

\section{REFERENCES}

1. Berson EL. Retinitis pigmentosa. The Friedenwald Lecture. Invest Ophthalmol Vis Sci. 1993; 34:1659-1676.

2. Hims MM, Diager SP, Inglehearn CF. Retinitis pigmentosa: genes, proteins and prospects. Dev Ophthalmol. 2003; 37:109-125.

3. Boughman JA, Conneally PM, Nance WE. Population genetic studies of retinitis pigmentosa. Am J Hum Genet. 1980; 32:223-235.

4. Jay $\mathrm{M}$. On the heredity of retinitis pigmentosa. Br J Ophthalmol. 1982; 66:405-416.

5. Haim M. Retinitis pigmentosa: problems associated with genetic classification. Clin Genet. 1993;44:62-70.

6. Wang Q, Chen Q, Zhao K, Wang L, Traboulsi EI. Update on the molecular genetics of retinitis pigmentosa. Ophthalmic Genet. 2001; 22:133-154.

7. Inglehearn CF. Molecular genetics of human retinal dystrophies. Eye (Lond). 1998; 12:571-579.

8. Lander ES, Botstein D. Homozygosity mapping: a way to map human recessive traits with the DNA of inbred children. Science. 1987; 236:1567-1570.

9. Sheffield VC, Stone EM, Carmi R. Use of isolated inbred human populations for identification of disease genes. Trends Genet. 1998; 14:391-396.

10. Kukita Y, Furuya H, Kobayashi T, Sakai N, Hayashi K. 
Characterization of the GALC gene in three Japanese patients with adult onset Krabbe disease. Genet Test. 1997; 1:217-223.

11. Schwarz JM, Cooper DN, Schuelke M, Seelow D. MutationTaster2: mutation prediction for the deepsequencing age. Nat Methods. 2014; 11:361-2.

12. Capriotti E, Fariselli P, Casadio R. I-Mutant2.0: predicting stability changes upon mutation from the protein sequence or structure. Nucleic Acids Res. 2005; 33:W306-10.

13. Richards S, Aziz N, Bale S, Bick D, Das S, Gastier-Foster J, Grody WW, Hegde M, Lyon E, Spector E, Voelkerding K, Rehm HL; ACMG Laboratory Quality Assurance Committee. Standards and guidelines for the interpretation of sequence variants: a joint consensus recommendation of the American College of Medical Genetics and Genomics and the Association for Molecular Pathology. Genet Med. 2015; 17:405-24.

14. Xu Y, Guan L, Shen T, Zhang J, Xiao X, Jiang H, Li S, Yang J, Jia X, Yin Y, Guo X, Wang J, Zhang Q. Mutations of 60 known causative genes in 157 families with retinitis pigmentosa based on exome sequencing. Hum Genet. 2014; 133:1255-71.

15. Yau KW, Baylor DA. Cyclic GMP-activated conductance of retinal photoreceptor cells. Annu Rev Neurosci. 1989; 12:289-327.

16. Körschen HG, Illing M, Seifert R, Sesti F, Williams A, Gotzes S, Colville C, Müller F, Dosé A, Godde M, Molday L, Kaupp UB, Molday RS, et al. A 240 kDa protein represents the complete beta subunit of the cyclic nucleotide-gated channel from rod photoreceptor. Neuron. $1995 ; 15: 627-636$.
17. Liu DT, Tibbs GR, Siegelbaum SA. Subunit stoichiometry of cyclic nucleotide-gated channels and effects of subunit order on channel function. Neuron. 1996; 16:983-90.

18. Zagotta WN, Siegelbaum SA. Structure and function of cyclic nucleotide-gated channels. Annu Rev Neurosci. 1996; 19:235-263.

19. Shammat IM, Gordon SE. Stoichiometry and arrangement of subunits in rod cyclic nucleotide-gated channels. Neuron. 1999; 23:809-819.

20. Kaupp UB. The cyclic nucleotide-gated channels of vertebrate photoreceptors and olfactory epithelium. Trends Neurosci. 1991; 14:150-157.

21. Kohl S, Baumann B, Broghammer M, Jagle H, Sieving P, Kellner U, Spegal R, Anastasi M, Zrenner E, Sharpe LT, Wissinger B. Mutations in the CNGB3 gene encoding the beta-subunit of the cone photoreceptor cGMP-gated channel are responsible for achromatopsia (ACHM3) linked to chromosome 8q21. Hum Mol Genet. 2000; 9:2107-2116.

22. Sundin OH, Yang JM, Li Y, Zhu D, Hurd JN, Mitchell TN, Silva ED, Maumenee IH. Genetic basis of total colour blindness among the Pingelapese islanders. Nat Genet. 2000; 25:289-293. 